\title{
Conhecer a complexidade do sistema climático para entender as mudanças climáticas
}

\author{
KNOWING THE COMPLEXITY OF THE CLIMATE SYSTEM tO UNDERSTAND CLIMATE CHANGE

\begin{abstract}
Ana Flávia Martins Montelro ${ }^{1}$, Ana Letícia Campos Yamamoto ${ }^{1}$, Paola do Nascimento Silva ${ }^{2}$, Michelle Simões Reboita ${ }^{3}$
1 - Doutoranda em Meio Ambiente e Recursos Hídricos pela Universidade Federal de Itajubá, Itaubá, Minas Gerais, Brasil.

2 - Mestranda em Meio Ambiente e Recursos Hídricos pela Universidade Federal de Itajubá, Itajubá, Minas Gerais, Brasil.

4 - Professora Associada da Universidade Federal de Itauubá, Itaubá, Minas Gerais, Brasil.

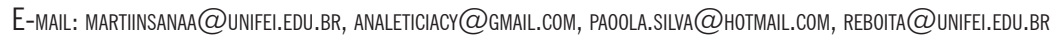

\begin{abstract}
The planet's temperature has been increasing since the beginning of the industrial revolution, as documented in the reports of the Intergovernmental Panel on Climate Change (IPCC). This affects the different components of the climate system, which in turn influence the climate (feedback mechanism). Therefore, to understand the physical processes associated with climate change, it is necessary to have a basic knowledge of the climate system, which is the focus of this study. The necessary knowledge is presented in simple language, so that the understanding of climate change can be acquired by all those with an interest.
\end{abstract}

Resumo: A temperatura do planeta vem apresentando aumento desde 0 início da revolução industrial, conforme documentado nos relatórios do Painel Intergovernamental sobre Mudanças Climáticas (IPCC). Isso afeta os diferentes componentes do sistema climático, que, por sua vez, influenciam 0 clima, em um mecanismo de retroalimentação. Para a compreensão dos processos físicos associados às mudanças climáticas é necessário um conhecimento básico sobre o sistema climático, que é o foco deste estudo. Apresentam-se aqui os conhecimentos necessários, em uma linguagem simples, para que o entendimento das mudanças climáticas seja adquirido por todos aqueles que tenham interesse.

\section{Introdução}

O clima do planeta Terra é resultado de um balanço de energia, que é basicamente a diferença entre a energia recebida do Sol e a emitida pela superfície (Brito Neto, 2017). Se a energia recebida exceder a emitida, o planeta se aqueceria; em contrapartida, se o planeta emitir mais do que receber, se esfriaria. Porém, o balanço de energia não acontece de forma tão simples, pois envolve uma série de processos que ocorrem na atmosfera, hidrosfera, criosfera, biosfera e litosfera. Esses são denominados de componentes do sistema climático e estão em constante interação trocando matéria e energia de diversas formas (IPCC, 2013).

O sistema climático é complexo; suas mudanças se refletem na história da humanidade. No passado ocorreram longos períodos (milhões de anos) em que grande parte da Terra foi coberta por gelo, sendo os períodos chamados de eras glaciais. Entre as
Citation/Citação: Monteiro, A. F. M., Yamamoto, A. L. C., Silva, P. N., \& Reboita, M. S. (2021). Conhecer a complexidade do sistema climático para entender as mudanças climáticas. Terræ Didatica, 17(Publ. Contínua), 1-12, e021006. doi: 10.20396/ td.v17i0.8663763

Keywords: Climate system components, IPCC, Physical processes, Energy budget, Earth's Climates.

Palavras-chave: Componentes do sistema climático, IPCC, Processos físicos, Balanço de energia, Climas terrestres.

\section{Manuscript/Manuscrito:}

Received/Recebido: 29/12/2020

Revised/Corrigido: 29/01/2021

Accepted/Aceito: 10/02/2021

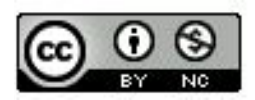

\begin{tabular}{c|c}
\hline C) Terrae Didat. & Campinas, SP \\
\hline
\end{tabular}

eras glaciais ocorrem períodos interglaciais, em que a temperatura terrestre é mais elevada (Leite, 2015). As alternâncias/mudanças no clima podem estar relacionadas a diferentes fatores como, por exemplo, ao movimento das placas tectônicas, composição atmosférica, variações da atividade solar e vulcânica, alteração nos parâmetros orbitais da Terra e nas correntes oceânicas (Hartmann, 1994, Galaz et al., 2018, Carneiro et al., 2019).

A atmosfera é composta por uma combinação de gases, sendo alguns classificados como gases de efeito estufa. Como será apresentado na próxima seção, os gases de efeito estufa existem desde a formação do planeta e contribuem para que a temperatura média do ar seja apta à vida. No entanto, a partir da Revolução Industrial, as atividades humanas passaram a contribuir para o aumento da concentração desses gases, acarretando a elevação da temperatura média do planeta (IPCC, 2013). Essa alteração, por sua vez, 
impacta os outros componentes do sistema climático, como por exemplo, a hidrosfera é afetada pelo aumento da temperatura do oceano e do nível do mar; a criosfera pelo degelo; a atmosfera apresenta eventos extremos como a intensificação nas ondas de calor, maiores períodos de seca e maior frequência de eventos extremos de chuva (INCT, 2014).

Dentre os gases de efeito estufa, o $\mathrm{CO}_{2}$ é o mais emitido pelas atividades antrópicas, sendo produzido, por exemplo, pela respiração, queima de combustíveis fósseis, atividade industrial e desmatamento. $\mathrm{O}$ aumento da concentração dos gases de efeito estufa afeta todos os componentes do sistema climático levando a mudanças no clima. Em termos simples, tais mudanças significam uma alteração no valor da temperatura média do planeta. Diante dessa abordagem, o estudo tem como objetivo descrever os componentes do sistema climático e suas interações para clarificar as causas das mudanças climáticas.

\section{Componentes do Sistema Climático}

\section{Atmosfera}

A atmosfera é uma camada que envolve a Terra, composta por gases e material particulado, e que se torna menos densa à medida que a distância da superfície terrestre aumenta (Fig. 1a). Não há uma altura limite que define o topo da atmosfera, os constituintes não se perdem no espaço devido à atração gravitacio- nal da Terra (NWS, 2020a). Os gases presentes na atmosfera podem ser classificados como permanentes ou de concentrações variáveis, sendo que os gases permanentes, nitrogênio $(\mathrm{N} ; 78 \%)$, oxigênio $\left(\mathrm{O}_{2}\right.$; $20,90 \%$ ) e argônio ( $\mathrm{Ar} ; 0,90 \%$ ), correspondem a cerca de 99,8\% da composição atmosférica (Latorre et al., 2002). Alguns dos gases com concentração variável são: dióxido de carbono $\left(\mathrm{CO}_{2}\right)$, ozônio $\left(\mathrm{O}_{3}\right)$ e vapor d'água $\left(\mathrm{H}_{2} \mathrm{O}(\mathrm{v})\right)$. Embora em uma concentração pequena, esses gases são fundamentais para a manutenção da vida, pois contribuem para o chamado efeito estufa, que é a absorção de radiação infravermelha emitida pela superfície e a reemissão para esta. Sem esse efeito natural, a temperatura média global seria de $-18^{\circ} \mathrm{C}$, que é quase duas vezes inferior à temperatura média global $\left(15^{\circ} \mathrm{C}\right)$ com a ocorrência desse efeito (Hartmann, 1994). O problema que se enfrenta com o efeito estufa na atualidade é que as ações antrópicas têm contribuído no aumento da concentração desses gases $\left(\mathrm{H}_{2} \mathrm{O}(\mathrm{v}), \mathrm{CO}_{2}, \mathrm{CH}_{4}, \mathrm{~N}_{2} \mathrm{O}, \mathrm{CFCs}\right.$ e $\left.\mathrm{O}_{3}\right)$ na atmosfera, o que acaba intensificado o efeito e levando a um aumento da temperatura média global.

A temperatura da atmosfera varia com a altura e, devido a essa variação, tem-se um parâmetro de classificação das camadas. As camadas da atmosfera são: troposfera, estratosfera, mesosfera, termosfera e exosfera (Fig. 1b). As camadas de transição entre elas possuem o sufixo "pausa", por exemplo, a camada de transição entre a troposfera e a estratosfera é a tropopausa (Dias et al., 2007, Ynoue et al., 2017).
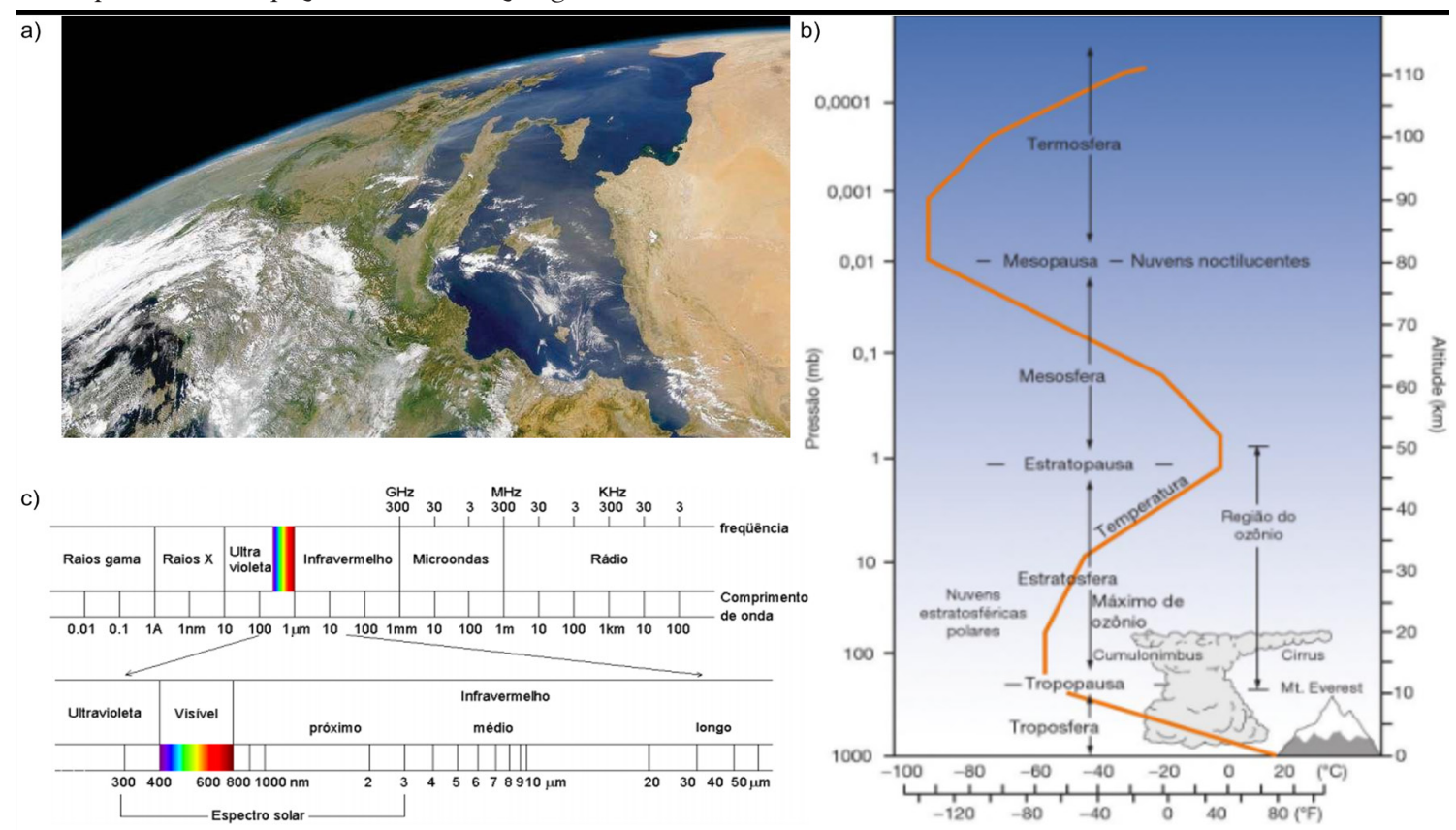

Figura 1. (a) Atmosfera vista do espaço (fina região azul ao longo da borda da Terra). Fonte: Ahrens et al. (2012); (b) Perfil vertical da atmosfera. Fonte: Barry \& Chorley (2010); (c) Espectro eletromagnético. Fonte: Dornelles (2008)

\begin{tabular}{c|c|c|c|c|c}
\hline C Terrae Didat. & Campinas, SP & v.17 & $1-12$ & $\mathrm{e} 021006$ & 2021 \\
\hline
\end{tabular}


A troposfera é a camada atmosférica mais próxima da superfície e possui cerca de $20 \mathrm{~km}$ de extensão vertical no equador e $8 \mathrm{~km}$ nos pólos. A extensão média é de $11 \mathrm{~km}$ (Dias et al., 2007). A troposfera contém $75 \%$ da massa total da atmosfera, incluindo todo o vapor d'água, e é a região onde a maioria dos fenômenos atmosféricos ocorrem. Nessa camada, a temperatura reduz à medida que a altitude cresce em uma taxa média de $6,5^{\circ} \mathrm{C} / \mathrm{km}$ até a tropopausa, onde a temperatura é cerca de $-55^{\circ} \mathrm{C}$ (Wallace \& Hobbs, 2007, Barry \& Chorley, 2010).

A estratosfera estende-se desde a tropopausa até aproximadamente $50 \mathrm{~km}$ de altitude. Contém cerca de $10 \%$ da massa total da atmosfera e a sua temperatura aumenta conforme o aumento da altitude (de -50 a $10^{\circ} \mathrm{C}$ ), sendo os maiores valores encontrados na estratopausa. Nessa camada, a concentração de vapor d'água é muito baixa e o gás predominante é o $\mathrm{O}_{3}$. Esse gás é o principal responsável pelo aquecimento da estratosfera, pois absorve radiação ultravioleta (uma das radiações mais energéticas emitidas pelo Sol), contribuindo para o aumento da temperatura (Dias et al., 2007, Barry \& Chorley, 2010), mas o aumento não atinge valores positivos. Os aviões voam na baixa estratosfera a fim de evitar a turbulência presente na troposfera (NOAA, 2016).

A mesosfera é a camada atmosférica mais fria, podendo chegar a $-133^{\circ} \mathrm{C}$, e possui comportamento similar ao da troposfera, isto é, redução da temperatura com a altitude (Dias et al., 2007, Barry \& Chorley, 2010). Nessa camada ocorre a queima da maioria dos meteoros, causando o efeito de rastro (Salby, 1996, NOAA, 2016).

A termosfera encontra-se acima da mesopausa e se estende por aproximadamente $600 \mathrm{~km}$, sendo que nessa camada a temperatura volta a aumentar (CSI, 2020). A parte inferior da termosfera é composta principalmente por nitrogênio e oxigênio nas formas molecular $\left(\mathrm{O}_{2}\right)$ e atômica $(\mathrm{O})$ (Barry \& Chorley, 2010). Devido à absorção de radiação de onda curta $(\lambda$ $\sim 200 \mathrm{~nm}$ ) nessa camada, a temperatura pode chegar a $1200{ }^{\circ} \mathrm{C}$, porém, como o ar é bastante rarefeito e a quantidade de moléculas é pequena, a transferência de calor é muito baixa (Dias et al., 2007).

A exosfera é a parte superior da termosfera e se estende até $10.000 \mathrm{~km}$ acima da superfície terrestre (CSI, 2020). Como o ar é extremamente rarefeito nessa altitude, a colisão entre moléculas é muito rara. Também é nessa região que os satélites orbitam a Terra (Dias et al., 2007).

Uma vez apresentada a estrutura vertical da atmosfera e sua composição química, é importante voltar à questão do efeito estufa. Para isso, inicia-se com a explicação da absorção de energia solar: o Sol emite energia em diferentes comprimentos de onda do espectro eletromagnético (Fig. 1c). As ondas curtas são as mais energéticas (raio gama, raio x e ultravioleta), enquanto as ondas longas são menos energéticas, como por exemplo a infravermelha. A maior parte da energia emitida pelo Sol é na faixa do visível $(500 \mathrm{~nm})$, porém os constituintes da atmosfera não são aptos a absorverem esse comprimento de onda. Por isso, é dito que a atmosfera é uma janela para a radiação na faixa do visível (300 a 900 nm; AMS, 2012). Então, como ocorre o aquecimento da troposfera? A energia que é recebida aquece primeiramente a superfície terrestre e essa camada por intermédio dos mecanismos de condução, convecção, turbulência e radiação, aquece as camadas de ar acima. Por motivos de brevidade, os processos não serão explicados aqui, mas o leitor encontra uma descrição detalhada em Reboita et al. (2012). A energia emitida pela superfície é em sua maior parte no comprimento de onda do infravermelho (ondas longas). Os gases de efeito estufa são absorvedores desse comprimento de onda, assim, absorvem a energia e emitem para a superfície (Fig. 2). O processo recebe o nome de efeito estufa, o qual esclarece o aquecimento da superfície terrestre pelo recebimento de energia em ondas curtas e longas. Portanto, quanto maior for a concentração de gases de efeito estufa na atmosfera, maior será o efeito de absorção da energia de onda longa e de emissão para a superfície, resultando no aumento da temperatura global (NOAA, 2020a). Uma vez que a temperatura média global é alterada (o valor médio é modificado), tem-se o que se chama de mudança climática.

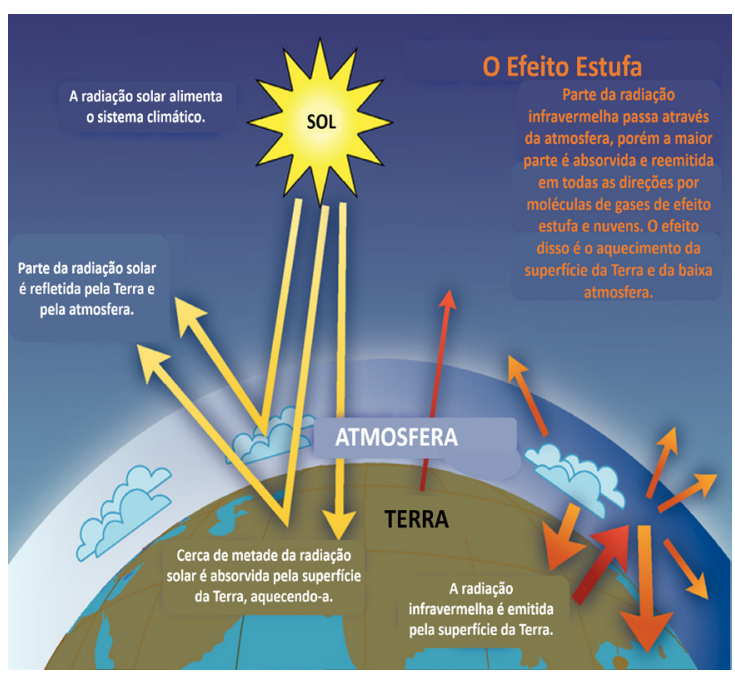

Figura 2. Esquema do efeito estufa. Fonte: Adaptado de IPCC (2007) 


\section{Hidrosfera}

A hidrosfera corresponde a água na fase líquida no planeta e compreende as águas oceânicas e continentais. A cada ano, os oceanos evaporam cerca de $502.800 \mathrm{~km}^{3}$ de água que retorna para a superfície terrestre por meio de precipitação (Lima, 2001). Do total de água encontrado na Terra, 97\% são águas salinas e apenas 3\% (35 milhões $\mathrm{km}^{3}$ ) são águas doces (Tundisi, 2003; Fig. 3a).

O ciclo hidrológico (Fig. 3b) equivale ao movimento da água no planeta entre as componentes do sistema climático e depende das peculiaridades de cada local (Kuchment, 2004). Os fatores que impulsionam o ciclo são: (a) energia térmica solar, (b) força do vento - que transporta vapor d'água na atmosfera de um local a outro e, (c) força de gravidade - que contribui para a precipitação, infiltração e deslocamento das massas de água (Tundisi, 2003). Há diversos processos envolvidos no ciclo hidrológico, porém os mais importantes são: evaporação, transpiração, condensação, precipitação e escoamento (NWS, 2020b). uma planta, apenas $1 \%$ é utilizado no processo de seu crescimento, os outros $99 \%$ são transferidos para a atmosfera na forma de vapor d'água (NWS, 2020b). A condensação é a mudança do estado de vapor d'água para estado líquido e, ocorre, em geral, devido ao resfriamento da massa de ar. A condensação é o processo que forma as nuvens (NWS, 2020b). Quando as nuvens precipitam, a água retorna para a superfície terrestre (parte da água precipitada pode ainda evaporar sem chegar à superfície). A precipitação pode ocorrer na forma líquida (chuva) e sólida (granizo, neve). Antes de atingir a superfície, uma parte da precipitação pode ser interceptada pela vegetação e/ou evaporada, e outra infiltra no solo. A água infiltrada, e que não é utilizada pela vegetação, percola pelo solo, fluindo para os reservatórios subterrâneos. $\mathrm{O}$ volume da infiltração depende das propriedades do solo e do seu teor de umidade (Kuchment, 2004). Quando a precipitação é excessiva e o solo já se encontra saturado, há o escoamento. Os rios e lagos são
A evaporação é o processo de mudança de fase da água disponível na superfície no estado líquido para o estado gasoso. A mudança de fase ocorre devido ao aumento da energia cinética das moléculas (Rodrigues, 2017). A água durante a evaporação é transferida da superfície (continental ou oceânica) para a atmosfera (NWS, 2020b) e pode ou não retornar para o mesmo local de onde foi evaporada, pois os ventos transportam o vapor d'água na atmosfera para locais distantes da fonte. A transpiração é o processo de evaporação das plantas e é controlado pela umidade da atmosfera e do solo na maioria das plantas. Do total de água transpirada por

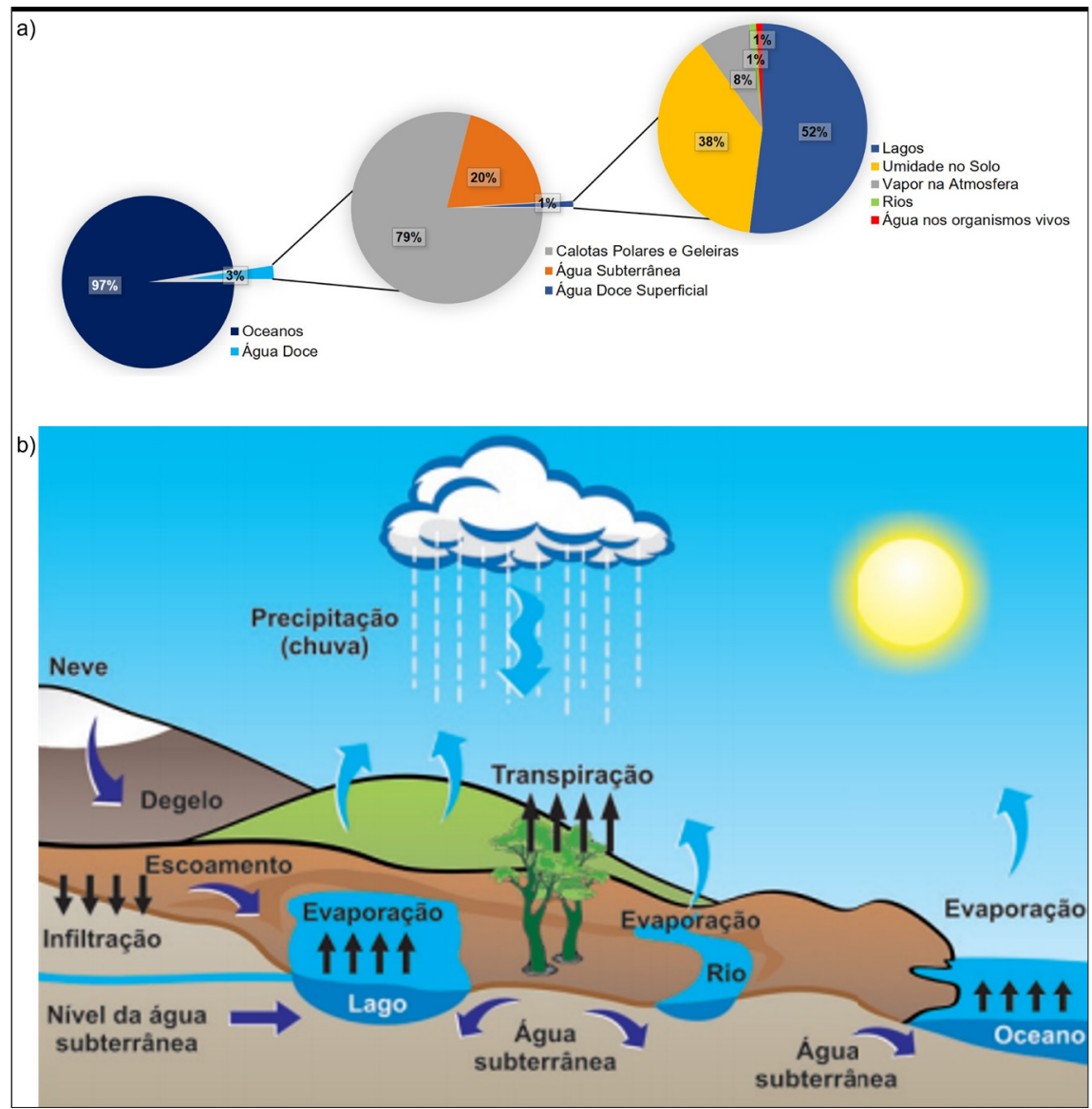

Figura 3. (a) Distribuição de água no planeta Terra. Fonte: Adaptado de Gomes et al. (2008); (b) Ciclo hidrológico. Fonte: Oliveira et al. (2009)

(C) Terrae Didat. 
resultados do escoamento superficial e são os meios de retorno da água para os oceanos. Assim, a evaporação do escoamento para a atmosfera reinicia o ciclo hidrológico (NWS, 2020b).

$\mathrm{Na}$ superfície terrestre, os oceanos são considerados os maiores reservatórios de gás carbônico, atuando como um sumidouro gigante que armazena esse gás por longos períodos em suas profundezas (NOAA, 2020b). Os rios, o escoamento de água subterrânea e a precipitação atmosférica são mecanismos de entrada do carbono nos ecossistemas marinhos (Souza et al., 2012); além disso, os organismos marinhos, como animais e plantas, produzem carbono durante a vida e na morte (NOAA, 2020b). O CO $\mathrm{CO}_{2}$ atua como importante gás do efeito estufa devido a sua capacidade de absorver radiação e, por consequência, promover o aumento da temperatura da superfície terrestre, tornando-a possível de ser habitada. Com o aumento da concentração do $\mathrm{CO}_{2}$ decorrentes de atividades antrópicas, ocorre a intensificação do efeito estufa, resultando em uma maior absorção de calor pela atmosfera e oceanos. Devido às propriedades térmicas da água, com o aumento na absorção de energia, os oceanos estão vivenciando uma expansão térmica, o que causa elevação no nível médio do mar. De 2005 até 2015, a média global do nível do mar aumentou a uma taxa de 3,6 mm/ano. É provável que até o ano de 2100 , se as emissões dos gases de efeito estufa não forem mitigadas, o nível do mar suba de 0,61 a 1,10 m (IPCC, 2019).

\section{Criosfera}

A criosfera (Fig. 4a) é a componente do sistema climático formada pela água no estado sólido (calotas polares, cobertura de neve, cristais de gelo na atmosfera, geleiras, gelo marinho, fluvial e lacustre, neve, permafrost (gelo no subsolo), solos

a)

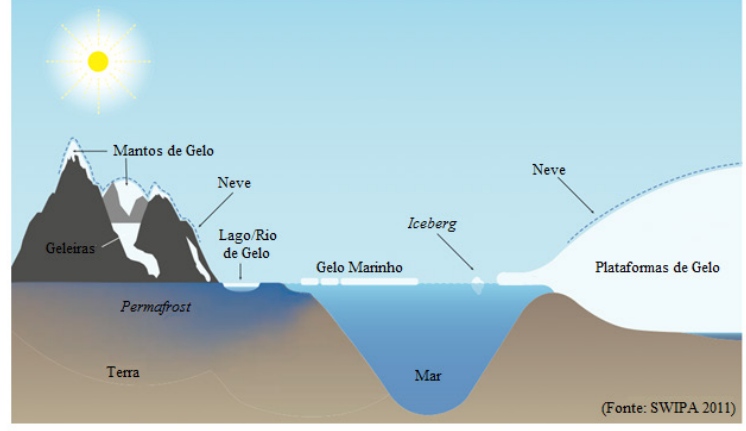

congelados e, principalmente, de dois mantos de gelo - Antártica e Groenlândia) (Brito Neto, 2017; Fig. 4b). A escala de tempo em que cada um dos componentes da criosfera se altera é diferente e todos estão diretamente relacionados às alterações climáticas, principalmente com relação às mudanças na temperatura e precipitação (IPCC, 2013). Portanto, a criosfera é considerada um medidor natural do sistema climático, interferindo, também, nos demais componentes do sistema (IPCC, 2013). Em resposta às alterações climáticas, os ecossistemas dentro da criosfera e aqueles que dependem dela estão em constante mudança (Fountain et al., 2012, Boetius et al., 2015). Ademais, as alterações provocadas na criosfera impactam os sistemas físicos, biológicos e sociais como um todo.

Nas eras passadas, o gelo permanente chegou a cobrir cerca de $30 \%$ da superfície do planeta e, atualmente, a relação reduziu-se para aproximadamente 10\% (INCT, 2014). As camadas de gelo contêm o melhor registro paleoclimático, tornando-se referência para avaliação das variações climáticas. Nas últimas décadas, drásticas mudanças foram detectadas nas áreas do planeta cobertas por gelo e neve (Levitus et al., 2001), tais como: derretimento do permafrost, desintegração das plataformas de gelo, diminuição da espessura das geleiras, redução da área de gelo marinho e variações na cobertura de neve (Fountain et al., 2012, Boetius et al., 2015). Com o conhecimento das diferentes forçantes que controlaram o clima no passado, a ciência é capaz de compreender os componentes que atualmente regulam o sistema climático.

A distribuição de gelo sobre a Terra é importante pois influencia a altura do nível do mar, balanço de energia, ciclo da água e formação de massas de água nas regiões polares (Brito Neto, 2017). A diminuição da criosfera pode ser relacionada a uma

b)

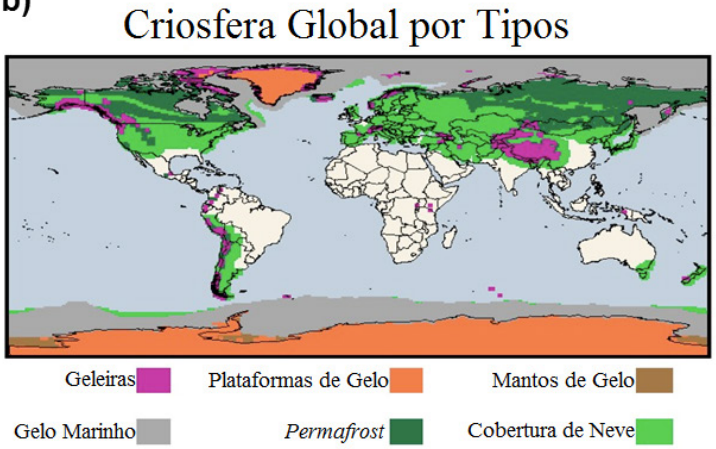

Figura 4. (a) Componentes gerais da criosfera; (b) Divisão global dos componentes da criosfera especificado por tipo. Fonte: globalcryospherewatch.org

\begin{tabular}{c|c}
\hline (C) Terrae Didat. & Campinas, SP \\
\hline
\end{tabular}


redistribuição da água sólida em suas fases líquidas e gasosas em escala planetária, devido ao aumento da temperatura média global em cenários futuros de mudanças climáticas. Dessa forma, causa impacto desde a seleção, adaptação e diversificação dos microorganismos (Boetius et al., 2015), até mudanças no balanço do sistema climático, por exemplo, no albedo, que indica a reflexão de uma superfície (Fountain et al., 2012). Quanto maior o albedo, menos energia é absorvida; isto é, neve e camadas brancas de gelo têm um alto albedo, refletindo com eficiência a radiação solar. Uma vez que o degelo reduz o fator de refletividade, isso leva a uma maior absorção de energia, alterando o balanço de energia da Terra e, consequentemente, a amplificação das mudanças climáticas (Pistone et al., 2014).

\section{Litosfera}

A litosfera é a camada superficial sólida do planeta Terra composta pela crosta terrestre (25-100 $\mathrm{km}$ de espessura) e oceânica (cerca de 5-10 km de espessura; Cruz et al., 2005) (Fig. 5). A composição mineralógica é majoritariamente formada por silício, alumínio e magnésio. Já, em termos de Geologia, é formada por rochas sedimentares, metamórficas e ígneas (Moreno \& Freitas, 2009). As rochas sedimentares são aquelas formadas por meio de processos físicos, químicos e biológicos de deposição de sedimentos (areia, argila, fragmentos de rochas) ou depósitos orgânicos (Gallegos, 1995). As rochas metamórficas, por sua vez, formam-se a partir de transformações físicas e químicas, provocadas por alterações de pressão e temperatura, de rochas pré-existentes (Orozco-Centeno, 2014). Já as rochas ígneas são formadas a partir da solidificação do magma, após seu resfriamento (Llambías, 2001).

A litosfera possui uma característica dinâmica e está em constante transformação devido à ação de agentes exógenos ou endógenos. Os agentes exógenos são caracterizados pela ação do vento, água, Sol e seres vivos, e favorecem a ocorrência de sedimentação, erosão e intemperismo (Ross, 2005). Por outro lado, as transformações por fatores endógenos como o tectonismo (movimentos das placas tectônicas), podem ocasionar os abalos sísmicos, tsunamis, atividades vulcânicas ou formações geológicas (Godoy, 2011).

A compreensão do comportamento e da dinâmica da litosfera, bem como suas características e composição é de grande relevância para a ativida-

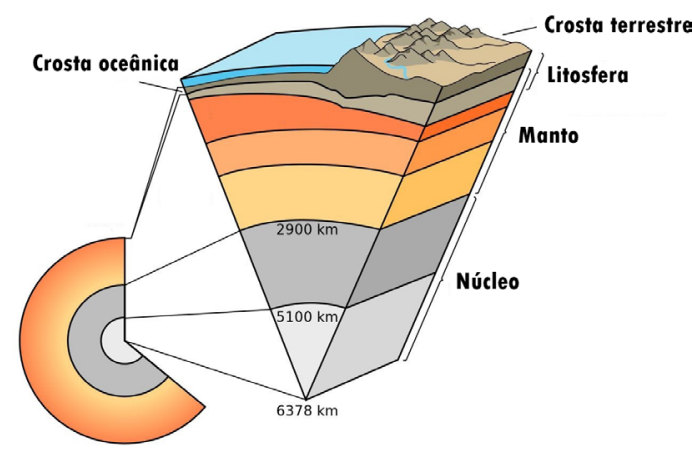

Figura 5. Representação da litosfera. Fonte: https://www. nationalgeographic.org (Adaptado)

de humana, pois é o local onde são desenvolvidas atividades de extração de minerais e aquelas relacionadas ao uso e ocupação do solo, que, de forma indireta, afetam a distribuição de energia no sistema climático (Barrón, 2009).

\section{Biosfera}

A biosfera (Fig. 6a) é a componente do sistema climático que compreende os ecossistemas e organismos vivos (biosfera terrestre e oceânica), interligados no meio abiótico (Barrón, 2009). A interação com os demais componentes do sistema climático (litosfera, atmosfera, criosfera e hidrosfera) é responsável pela manutenção da vida na Terra, e o conhecimento dessa interação é fundamental para a previsão da evolução do clima no planeta (Dias et al., 2005). Por ser um sistema dinâmico, as transformações que ocorrem na biosfera acarretam processos de ciclagem e redistribuição de elementos químicos por meio de interações entre os meios biótico e abiótico, denominados de ciclos biogeoquímicos (Summons, 1993). Os ciclos estão relacionados com os processos geológicos, hidrológicos e biológicos, e são fundamentados na transformação de substâncias inorgânicas a orgânicas (e vice-versa) (González et al., 2007). O estudo é essencial para a compreensão das conexões entre componentes do sistema climático e a dinâmica dos ecossistemas (Esteve \& Jaén, 2013). A seguir, é apresentada uma breve descrição dos ciclos biogeoquímicos do carbono e nitrogênio.

\section{Ciclo do carbono}

O carbono é um elemento essencial para a existência de vida na Terra, sendo a base de moléculas orgânicas de proteínas, carboidratos, lipídeos e ácidos nucleicos (Souza et al., 2012). Na natureza, se apresenta na forma orgânica, em 
componentes no oceano, solo e rochas sedimentares, e na forma inorgânica, como compostos produzidos pelo metabolismo de organismos (Enrich-Prast et al., 2018). Os principais reservatórios de carbono são a atmosfera, o oceano e a superfície terrestre, incluindo carbono orgânico encontrado no solo (Post et al., 1990), e é por meio desses reservatórios que o ciclo ocorre (Fig. 6b). O carbono, na forma de dióxido de carbono $\left(\mathrm{CO}_{2}\right)$, é absorvido da atmosfera e liberado na forma de oxigênio pelos organismos autótrofos no processo conhecido como fotossíntese. Após ser assimilado pelos seres vivos, o retorno de $\mathrm{CO}_{2}$ para a atmosfera é dado pela respiração e decomposição de matéria orgânica morta. No oceano, o fluxo de carbono é regulado pela atividade fotossintética do fitoplâncton e pelo processo de dissolução e de liberação de $\mathrm{CO}_{2}$ na água (Aduan et al., 2004). A adição de $\mathrm{CO}_{2}$ à água do mar ocorre devido à troca entre a atmosfera $\mathrm{e}$ o oceano, por difusão molecular. Esse processo acarreta no aumento do $\mathrm{CO}_{2}$ dissolvido, que reage com a água para formar ácido carbônico $\left(\mathrm{H}_{2} \mathrm{CO}_{3}\right)$ (Schellnhuber et al., 2006). Parte deste ciclo vêm sofrendo modificações principalmente em razão da ação humana: emissão de combustíveis fósseis, queimadas e desmatamento. Esses fatores têm contribuído para o aumento da concentração de $\mathrm{CO}_{2}$ na atmosfera, influenciando diretamente a ciclagem do elemento (Bustamante et al., 2017).

\section{Ciclo do nitrogênio}

O gás nitrogênio $\left(\mathrm{N}_{2}\right)$ é o elemento mais abundante na atmosfera terrestre, ocupando aproximadamente $78 \%$ do volume total da atmosfera seca (ausência de vapor d'água) (Ynoue et al., 2017). É fundamental para os seres vivos, uma vez que é parte integrante de enzimas, proteínas e ácidos nucleicos (Aduan et al., 2004, Denk et al., 2017). Apesar de sua disponibilidade, o $N_{2}$ não pode ser assimilado diretamente pela maioria dos organismos vivos devido à tripla ligação covalente entre seus átomos, sendo necessário convertê-lo na forma inorgânica mineral como amônia $\left(\mathrm{NH}_{3}\right)$, amônio $\left(\mathrm{NH}_{4}^{+}\right)$ou nitrato $\left(\mathrm{NO}_{3}^{-}\right)$, pelos processos de mineralização (ou amonificação) e fixação (García-Velázquez \& Gallardo, 2017, Vieira, 2017). No primeiro processo, o nitrogênio entra no ciclo biológico pela mineralização da matéria orgânica em $\mathrm{NH}_{3}$ ou $\mathrm{NH}_{4}^{+}$, a partir da atividade microbiana (Rincón \& Gutiérrez, 2012). Já, na fixação, o
$\mathrm{N}_{2}$ é transformado em $\mathrm{NH}_{3}$ ou $\mathrm{NH}_{4}^{+}$majoritariamente por microorganismos de vida livre (em lagos, solos e sedimentos) ou associado às raízes de plantas, como as leguminosas (Enrich-Prast et al., 2018). Após o processo, o amônio ainda pode ser oxidado para nitrito $\left(\mathrm{NO}_{2}^{-}\right)$e, consequentemente, para nitrato, na presença de oxigênio, por microorganismos quimioautotróficos no processo denominado de nitrificação. Assim, quando liberados para o solo, os compostos inorgânicos de nitrogênio podem ser absorvidos e metabolizados por produtores primários (Vieira, 2017). Por fim, as formas oxidadas de nitrogênio (nitrito e nitrato) são reduzidas a $\mathrm{N}_{2}$ e óxido nitroso $\left(\mathrm{N}_{2} \mathrm{O}\right)$ por microorganismos em baixas condições aeróbicas ou anaeróbicas, e retornam para a atmosfera no processo de desnitrificação (Avila et al., 2002, Duarte, 2006) (Fig. 6c).

a)

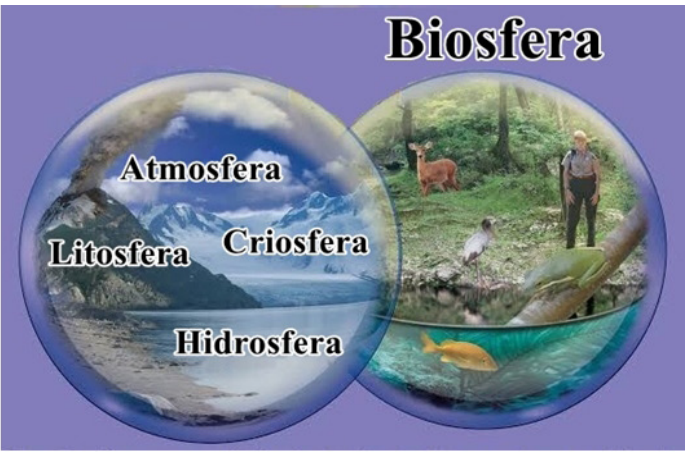

b)

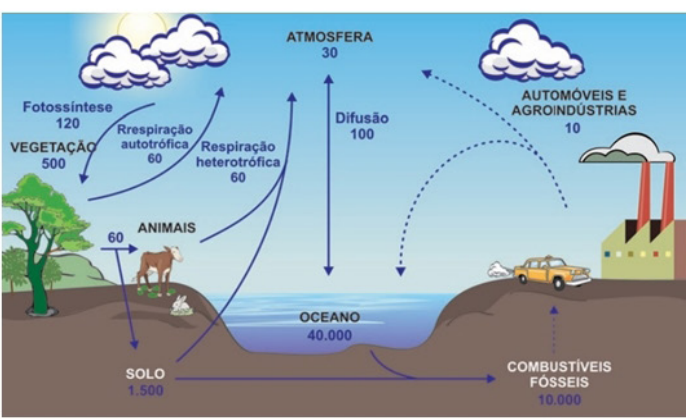

c)

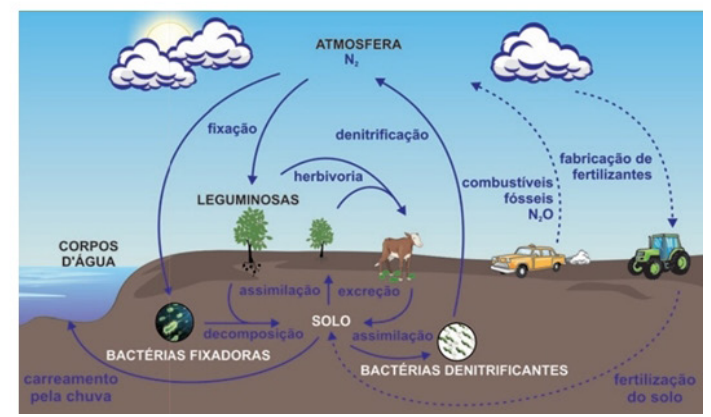

Figura 6. (a) Representação da biosfera e demais componentes do sistema climático (Fonte: https://pubs. usgs.gov - Adaptado); (b) Ciclo do carbono (Fonte: Oliveira et al., 2009); (c) Ciclo do nitrogênio (Fonte: Oliveira et al., 2009) 


\section{Mecanismos de Retroalimentação (feedback)}

O sistema climático é impulsionado por um grande número de processos e interações que ocorrem entre seus componentes (atmosfera, hidrosfera, criosfera, biosfera e litosfera) (Ambrizzi et al., 2019). Assim, uma perturbação no sistema pode acarretar um efeito nas componentes associadas. Esse processo é denominado de mecanismo de retroalimentação ou de feedback.

Os mecanismos de retroalimentação do sistema terrestre são importantes reguladores da dinâmica do clima tanto localmente como globalmente. Tais mecanismos podem ocasionar efeitos positivos ou negativos à medida que são transferidos de um subcomponente do sistema para outro (Ginzburg \& Demchenko, 2019). Os feedbacks positivos são responsáveis pela amplificação dos efeitos das forçantes radiativas, isto é, aumentam o aquecimento inicial e intensificam as alterações no clima. Já, os feedbacks negativos tendem a reduzir os efeitos das forçantes radiativas (Carslaw et al., 2010).

\section{Vapor d'água}

O mecanismo de feedback vapor d'água (Fig. 7a) é caracterizado por um efeito positivo, já que o vapor d'água é o principal gás de efeito estufa.
A elevação da concentração de $\mathrm{CO}_{2}$ na atmosfera acarreta o aumento da absorção de radiação infravermelha que é emitida para a atmosfera na forma de ondas longas pela Terra e sua emissão de volta para a superfície, o que aumenta, por sua vez, a temperatura do ar e, portanto, a capacidade de retenção de vapor d'água na atmosfera (a quantidade de vapor d'água que o ar pode manter está relacionado com a temperatura). Desta forma, há maior concentração de vapor d'água e intensificação do efeito estufa, amplificando a tendência de aquecimento em resposta a uma forçante inicial (Barbosa, 2014, Onça, 2014).

\section{Gelo-albedo}

O mecanismo de feedback gelo-albedo (Fig. 7b) é o processo associado às alterações na cobertura de gelo e neve (albedo) que resultam na tendência de aquecimento e/ou resfriamento (Curry et al., 1995). Se a temperatura aumenta, a extensão de gelo e neve diminui, o que reduz o albedo da superfície. Uma vez que o albedo do gelo diminui, uma maior quantidade de radiação solar é absorvida na superfície, o que acarreta o aumento da temperatura da superfície (feedback positivo). Por outro lado, se a temperatura diminui, aumentam-se as áreas com cobertura de gelo e neve, o que impacta diretamente no albedo a)
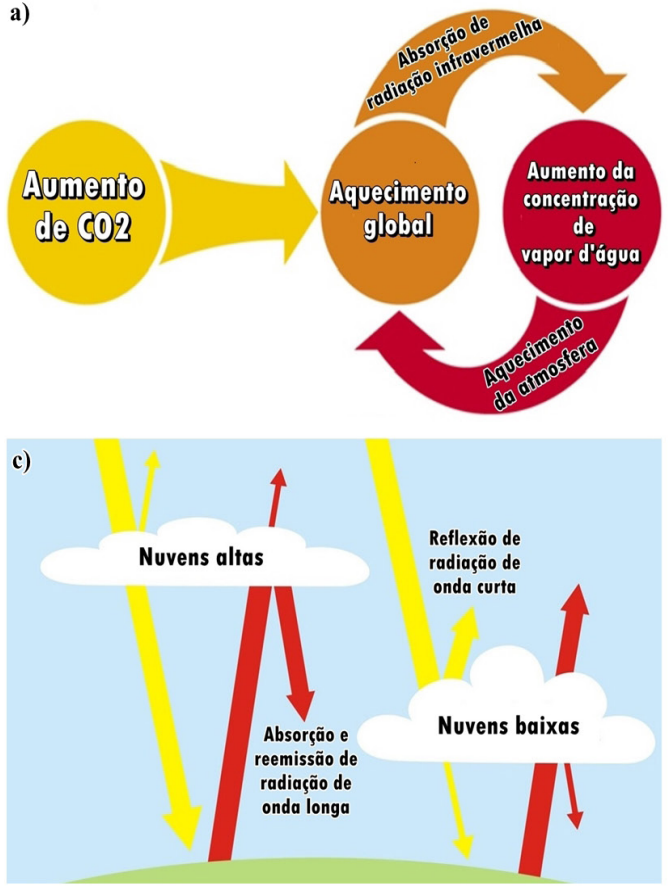

b)

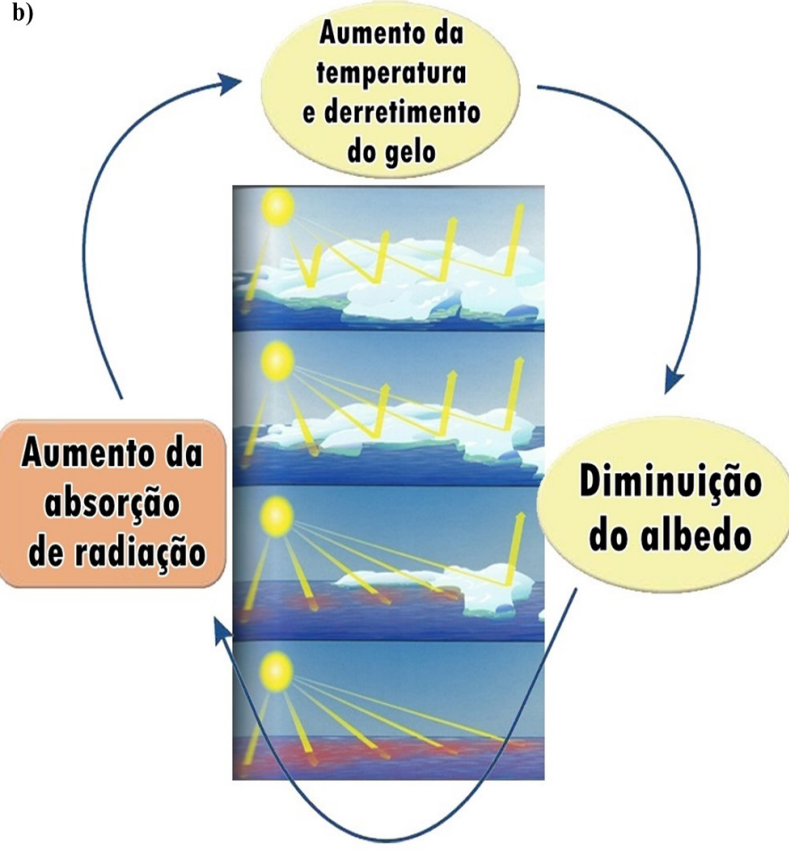

Figura 7. Mecanismos de retroalimentação: (a) vapor d'água (Fonte: https://www.e-education.psu.edu - Adaptado); (b) gelo-albedo (Fonte: http://www.onlyonesolution.org - Adaptado); (c) nuvens (Fonte: https://static.skepticalscience. com - Adaptado) 
e, consequentemente, mais radiação solar é refletida, reduzindo a temperatura da superfície (feedback negativo) (Qu \& Hall, 2007, Kashiwase et al., 2017).

\section{Nuvens}

O mecanismo de feedback das nuvens (Fig. 7c) é a maior fonte de incerteza na resposta climática, uma vez que a simulação das nuvens e seus processos radiativos têm sido um desafio para a modelagem numérica, pois envolvem processos físicos de microfísica (Gettelman \& Scherwood, 2016, Ceppi et al., 2017). As nuvens exercem um importante papel no clima do planeta, já que atuam no balanço de energia. No entanto, a magnitude desse efeito depende das características de altitude, cobertura, tipo, composição ou profundidade óptica (Hartmann et al., 1992; Chen et al., 2000). O consenso científico é o de que as nuvens baixas (abaixo de $2 \mathrm{~km}$ ) têm a característica de resfriar a temperatura do planeta devido à reflexão da radiação de onda curta (feedback negativo). Em contrapartida, as nuvens altas (acima de $6 \mathrm{~km}$ ), constituídas de gelo, absorvem a radiação de onda longa e reemitem à sua temperatura, contribuindo para a intensificação do efeito estufa (feedback positivo) (Yamasoe \& Corrêa, 2016).

A compreensão dos efeitos dos mecanismos de retroalimentação, no sistema climático, é uma ferramenta importante no entendimento do sistema frente às alterações do clima, visto que os mecanismos atuam em resposta a uma perturbação e para a estabilidade do mesmo.

\section{Mudanças Climáticas}

As mudanças climáticas constituem alterações nas condições do clima terrestre, tais como temperatura, precipitação, entre outros. Desde o início da era industrial tem sido observado um aumento da temperatura média global do ar e simultaneamente aumento do $\mathrm{CO}_{2}$. Em um observatório localizado na Ilha Grande do Havaí, no oceano Pacífico, mensuram-se os constituintes atmosféricos capazes de alterar o clima. O observatório, chamado Mauna Loa Observatory (MLO), teve suas atividades iniciadas na década de 1950, e atualmente é bastante conhecido pelas medições do aumento de concentração do $\mathrm{CO}_{2}$ na atmosfera. A quantidade de $\mathrm{CO}_{2}$ presente na atmosfera vem aumentando cada vez mais (Fig. 8), em 1960 a concentração era de aproximadamente 315 ppm, já em 2020 esse valor aumentou para quase 420 ppm (NOAA, 2020c).

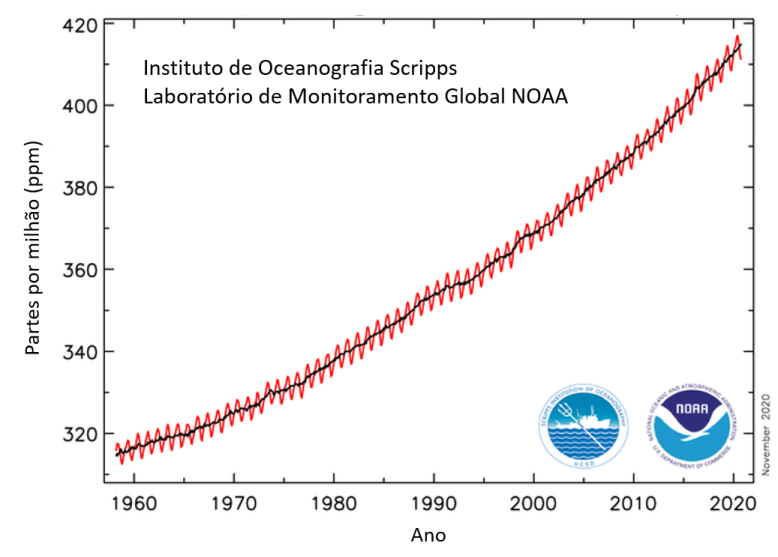

Figura 8. $\mathrm{CO}_{2}$ atmosférico no observatório de Mauna Loa. Fonte: Adaptado de NOAA (2020c)

Até aqui pode-se resumir que há fatores que alteram as forçantes climáticas (possibilidade de absorver mais ou menos energia) que, por sua vez, levam a mudanças na média climatológica de longo período da temperatura do ar e a distúrbios do clima refletidos na forma da ocorrência de ondas de calor mais intensas e frequentes; chuvas intensas que podem favorecer inundações e enchentes; aumento da frequência de ciclones tropicais; variações na frequência e intensidade de eventos tipo $E l$ Niño (Marengo, 2001), entre outros. A explicação caracteriza a expressão mudanças climáticas.

$\mathrm{O}$ texto focou a contribuição do homem em aumentar a concentração de gases de efeito estufa na atmosfera. Portanto, como o homem constitui um fator antrópico, aqui, são sumarizadas algumas das atividades antrópicas que mais contribuem para o aumento da concentração dos gases de efeito estufa na atmosfera:

- Processo de urbanização: devido à mudança no uso do solo para a construção de cidades, ocorre a alteração do fluxo de energia pela concentração de poluentes e partículas sólidas presentes. A quantidade de vegetação nas cidades é reduzida, portanto, a maior parte da energia absorvida pela vegetação é usada para aquecimento ao invés da evaporação. Já a área rural e de cultura agrícola absorve menos energia, com temperaturas menores do que as cidades. A diferença de temperatura entre os ambientes ocasiona as ilhas de calor urbano (Teodoro \& Amorim, 2008);

- Queima de combustíveis fósseis (petróleo, carvão e gás natural) para geração de energia, atividades industriais, transportes, entre outros; 
- Agropecuária: emite $\mathrm{CH}_{4}, \mathrm{CO}_{2}, \mathrm{CO}, \mathrm{N}_{2} \mathrm{O}$ e NOx, que causam aquecimento global (Lima, 2002);

- Queimadas: contribuem para aumentar o efeito estufa na atmosfera, devido à emissão de $\mathrm{CO}_{2}, \mathrm{CH}_{4}$ e NOx. As emissões provenientes de queimadas alteram o balanço radiativo da atmosfera pois os aerossóis presentes refletem e espalham a radiação solar de volta para o espaço, diminuindo a absorção pela superfície terrestre, e, absorvem a radiação, aquecendo a atmosfera (Freitas et al., 2005).

Os dois últimos relatórios do IPCC (2007, 2013) mostraram que se o fator antrópico não for adicionado nas simulações climáticas é impossível reproduzir o aumento da temperatura média global das últimas décadas só com base nos fatores naturais. Em relação aos fatores naturais tem-se, por exemplo: variação dos parâmetros orbitais da Terra, atividades vulcânicas, manchas solares, entre outros. Uma abordagem dos fatores é dada em Hartmann (1994), Teodoro e Amorim (2008), Reboita et al. (2015) e Ynoue et al. (2017).

\section{Considerações Finais}

Este estudo objetivou descrever os componentes do sistema climático e suas interações, focando nas alterações da forçante radiativa associada às atividades antrópicas. O balanço de energia, que é o mecanismo que dirige o clima do planeta Terra, envolve processos que ocorrem entre os diferentes componentes do sistema climático: atmosfera, biosfera, criosfera, hidrosfera e litosfera. Portanto, alterações em um desses componentes afetam os demais por intermédio dos mecanismos de feedback e podem causar mudanças no clima.

\section{Agradecimentos}

Os autores agradecem o auxílio financeiro da Coordenação de Aperfeiçoamento de Pessoal de Nível Superior (CAPES) Código 001, Fundação de Amparo à Pesquisa do Estado de Minas Gerais (FAPEMIG) e Conselho Nacional de Desenvolvimento Científico e Tecnológico (CNPq).

\section{Referências}

Aduan, R. E., Vilela, M. F., \& Reis Júnior, F. B. dos (2004). Os grandes ciclos biogeoquímicos do planeta. Embrapa Cerrados-Documentos (INFOTECA-E). URL: http://www.infoteca.cnptia.embrapa.br/infoteca/ handle/doc/569371. Acesso 03.11.2020.

Ahrens, C. D., Jackson, P. L., \& Jackson, C. E. J. (2012). Meteorology Today: an introduction to weather, climate, and the environment. First Canadian Edition: Cengage Learning. 640p.

Ambrizzi, T., Reboita, M. S., Rocha, R. P. da, \& Llopart, M. (2019). The state-of-the-art and fundamental aspects of Regional Climate Modeling in South America. Ann. N. Y. Acad. Sci., 1436(1), 98-120. doi: 10.1111/nyas. 13932.

AMS. American Meteorological Society. (2012). Atmospheric Window. URL: https://glossary.ametsoc.org/ wiki/Atmospheric_window. Acesso 05.12.2020.

Avila, J. P., Canul, R. P., \& Sansores, A. C. (2002). Análisis del ciclo del nitrógeno en el medio ambiente con relación al agua subterránea y su efecto en los seres vivos. Ingeniería, 6(3), 73-81.

Barbosa, H. M. J. (2014). Vapor de água na atmosfera: do efeito estufa às mudanças climáticas. Revista USP, (103), 67-80. doi: 10.11606/issn.2316-9036. v0i103p67-80.

Barrón, L. G. (2009). El sistema climático. In: Barrón, L. G. (Coord.), Murillo, P. G., Diña, V. J., González, J. M., Luna, J. N., \& Martín, A. S. Cambios climáticos y efectos ambientales. Sevilla: Universidad Internacional de Andalucía. p. 10-19.

Barry, R. G., \& Chorley, R. J. (2010). Atmosphere, weather and climate. 9 ed. USA: Routledge. 536p.

Boetius, A., Anesio, A. M., Deming, J. W., Mikucki, J. A., \& Rapp, J. Z. (2015). Microbial ecology of the cryosphere: sea ice and glacial habitats. Nat. Rev. Microbiol., 13(11), 677-690. doi: 10.1038/nrmicro3522.

Brito Neto, F. A. (2017). Análise do comportamento da criosfera nos períodos do início do Holoceno e pré-industrial. Natal: Universidade Federal do Rio Grande do Norte (UFRN). 30p. (Trab. Concl. Curso). URL: http://monografias.ufrn.br/handle/123456789/5464. Acesso 01.11.2020.

Bustamante, M., Ometto, J., \& Martinelli, L. A. (2017). Biodiversidade e ciclos Biogeoquímicos. In: Nobre, C. A., \& Marengo, J. A. (Orgs.). Mudanças climáticas em rede. Bauru: Canal 6. p. 189-201.

Carneiro, C. D. R., Franco, L. M., Freitas, G. B. F. de., \& Gonçalves, P. W. (2019). Processos de mudança climática durante a "Pequena Idade do Gelo" sob o enfoque de Ciência do Sistema Terra. Terræ Didatica, 15, 1-10, e019043. doi: 10.20396/td.v15i0.8657525.

Carslaw, K. S., Boucher, O., Spracklen, D. V., Mann, G. W., Rae, J. G. L., Woodward, S., \& Kulmala, M. (2010). A review of natural aerosol interactions and feedbacks within the Earth system. Atmospheric Chem. Phys., 10(4), 1701-1737. doi: 10.5194/acp-101701-2010.

Ceppi, P., Brient, F., Zelinka, M. D., \& Hartmann, D. L. (2017). Cloud feedback mechanisms and their representation in global climate models. Wiley Interdiscip. Rev. Clim. Change, 8(4), e465. doi: 10.1002/ wcc. 465.

Chen, T., Rossow, W. B., \& Zhang, Y. (2000). Radiative effects of cloud-type variations. J. Clim., 13(1), 264286. doi: $10.1175 / 1520-0442(2000) 013<0264:$ REO CTV>2.0.CO;2.

Cruz, F. N., Borba, G. L., \& Abreu, L. R. D. (2005). A Terra - litosfera e hidrosfera. Natal: EDUFRN (Editora

\begin{tabular}{c|c|c|c|c|c}
\hline (C) Terrae Didat. & Campinas, SP & v.17 & $1-12$ & e 021006 & 2021 \\
\hline
\end{tabular}


da UFRN). 348p.

CSI. Climate Science Investigations. (2020). Energy: The Driver of Climate. URL: http://www.ces.fau. edu/nasa/module-2/atmosphere/earth.php. Acesso 05.11 .2020

Curry,J.A.,Schramm,J.L., \&Ebert,E.E. (1995). Seaice-albedoclimate feedback mechanism.J. Clim., 8(2), 240247. doi: 10.1175/1520-0442(1995)008<0240:SIACFM $>2.0 . \mathrm{CO} ; 2$.

Denk, T. R., Mohn, J., Decock, C., Lewicka-Szczebak, D., Harris, E., Butterbach-Bahl, K., \& Wolf, B. (2017). The nitrogen cycle: A review of isotope effects and isotope modeling approaches. Soil Biol. Biochem. 105, 121-137. doi: 10.1016/j.soilbio.2016.11.015.

Dias, M. A. F. S., Cohen, J. C. P., \& Gandu, A. W. (2005). Interações entre nuvens, chuvas e a biosfera na Amazônia. Acta Amaz., 35(2), 215-222. doi: 10.1590/ S0044-59672005000200011.

Dias, A. A. C., Andrade-Neto, A. V., \& Miltão, M. S. R. (2007). A atmosfera terrestre: composição e estrutura. Caderno de Física da UEFS, 5, 21-40.

Dornelles, K. A. (2008). Absortância solar de superficies opacas: Métodos de Determinação e Base de Dados para Tintas Látex Acrílica e PVA. Campinas: Universidade Estadual de Campinas. Unicamp. 152p. (Tese Doutorado). URL: http://repositorio.unicamp.br/ jspui/handle/REPOSIP/257698. Acesso 06.11.2020.

Duarte, C. M. (Coord.), Alonso, S., Benito, G., Dachs, J., Montes, C., Pardo, M., Ríos, A. F., Simó, R., \& Valladares, F. (2006). Cambio global Impacto de la actividad humana sobre el sistema Tierra. Madrid: Consejo Superior de Investigaciones Científicas (CSIC). 167p.

Enrich-Prast, A., Gaxiola, A., Santoro, A. L., Durán, J., Rodríguez, A., \& Mariota, H. (2018). Ciclos biogeoquímicos y cambios globales. In: Marquet, P., Valladares, F., Magro, S., Gaxiola, A., \& Enrich-Prast, A. (Ed.). Cambio Global: una mirada desde Iberoamérica. Madrid: Consejo Superior de Investigaciones Científicas (CSIC). p. 111-125.

Esteve, P., \& Jaén, M. (2013). El papel de los ciclos biogeoquímicos en el estudio de los problemas ambientales en Educación Secundaria. Revista de Investigación en la Escuela, 80, 77-88. doi: 10.12795/IE.2013.i80.06.

Fountain, A. G., Campbell, J. L., Schuur, E. A., Stammerjohn, S. E., Williams, M. W., \& Ducklow, H. W. (2012). The disappearing cryosphere: impacts and ecosystem responses to rapid cryosphere loss. BioScience, 62(4), 405-41. doi: 10.1525/bio.2012.62.4.11.

Freitas, S. R., Longo, K. M., Silva Dias, M. A. F., \& Silva Dias, P. L. (2005). Emissões de queimadas em ecossistemas da América do Sul. Estud. Av., 19(53), 167185. doi: 10.1590/S0103-40142005000100011.

Galaz, V., Crona, B., Dauriach, A., Scholtens, B., \& Steffen, W. (2018). Finance and the Earth system - Exploring the links between financial actors and non-linear changes in the climate system. Glob. Environ. Change, 53, 296-302. doi: 10.1016/j.gloenvcha.2018.09.008.

Gallegos, J. A. (1995). La clasificación de las rocas sedimentarias: sugerencias para su aprendizaje. Enseñan$z a$ de las Ciencias de la Tierra, 3(3), 154-163.

García-Velázquez, L., \& Gallardo, A. (2017). El ciclo global del nitrógeno. Una visión para el ecólogo terrestre. Revista Ecosistemas, 26(1), 4-6. doi: 10.7818/ ECOS.2017.26-1.02.
Gettelman, A., \& Sherwood, S. C. (2016). Processes responsible for cloud feedback. Curr. Clim. Change Rep., 2(4), 179-189. doi: 10.1007/s40641-016-00528.

Ginzburg, A. S., \& Demchenko, P. F. (2019). Anthropogenic meso-meteorological feedbacks: A review of a recent research. Izv. Atmos.Ocean. Phy., 55(6), 573590. doi: 10.1134/S0001433819060045.

Godoy, D. F. de, Hackspacher, P. C., \& Genaro, D. T. (2011). Tectônica x sedimentação. In: Hackspacher, P. C. (Org.). Dinâmica do relevo: Quantificação de processos formadores. São Paulo: Editora Unesp. p. 27-51.

González, F. M., García, F. G., \& Tejada, M. P. J. (2007). Las ilustraciones de los ciclos biogeoquímicos del carbono y nitrógeno en los textos de secundaria. Revista Eureka sobre Enseñanza y Divulgación de las Ciencias, 4(3), 442-460.

Hartmann, D. L., Ockert-Bell, M. E., \& Michelsen, M. L. (1992). The effect of cloud type on Earth's energy balance: Global analysis. J. Clim., 5(11), 1281-1304. doi: 10.1175/1520-0442(1992)005<1281:TEOC$\mathrm{TO}>2.0 . \mathrm{CO} ; 2$.

Hartmann, D. L. (1994). Global Physical Climatology. USA: Academic Press. 411p.

INCT. Institutos Nacionais de Ciência e Tecnologia (da Criosfera). (2014). Compreendendo as mudanças do clima pela análise de testemunhos de gelo. URL: https://www.ufrgs.br/inctcriosfera/arquivos/Compreendendo\%20as\%20mudancas\%20climaticas.pdf. Acesso 03.11.2020.

IPCC. Intergovernmental on Climate Change. (2007). Climate Change 2007: The Physical Science Basis. Contribution of Working Group I to the Fourth Assessment Report of the Intergovernmental Panel on Climate Change [Solomon S., Qin D., Manning M., Marquis M., Averyt K., Tignor M. M. B., Miller JR., H. L., \& Chen Z]. United Kingdom and New York: Cambridge University Press.

IPCC. Intergovernmental on Climate Change. (2013). Climate Change 2013: The Physical Science Basis. Contribution of Working Group I to the Fifth Assessment Report of the Intergovernmental Panel on Climate Change, Cambridge, New York: Cambridge University Press.

IPCC. Intergovernmental on Climate Change. (2019). Summary for Policymakers. In: IPCC Special Report on the Ocean and Cryosphere in a Changing Climate [Potner, H. O., Roberts, D. C., Masson-Delmotte, V., Zhai, P., Tignor, M., Poloczanska, E., Mintenbeck, K., Nicolai, M., Okem, A., Petzold, J., Rama, B., \& Weyer, N. (Eds.)]. (In press).

Kashiwase, H., Ohshima, K. I., Nihashi, S., \& Eicken, H. (2017). Evidence for ice-ocean albedo feedback in the Arctic Ocean shifting to a seasonal ice zone. Sci. Rep., 7(1), 1-10. doi: 10.1038/s41598-017-08467-z.

Kuchment, L.S. (2004). The hydrological cycle and human impact on it. In: Hoekstra, A. Y., \& Savenije, H. H.G. (Eds.). Water Resources Management. Oxford: Eolss Publishers.

Latorre, M. L., Carvalho Júnior, O. A. D., Carvalho, A. P. F. D., \& Shimabukuro, Y. E. (2002). Correção atmosférica: conceitos e fundamentos. Espaç. Geogr., 5(1), 153-178.

Leite, J. C. (2015). Do mistério das eras do gelo às mudanças climáticas abruptas. Sci. Stud., 13(4), 811-

\begin{tabular}{c|c|c|c|c|c}
\hline (C) Terrae Didat. & Campinas, SP & v.17 & $1-12$ & $\mathrm{e} 021006$ & 2021 \\
\hline
\end{tabular}


839. doi: 10.1590/S1678-31662015000400005.

Levitus, S., Antonov, J. I., Wang, J., Delworth, T. L., Dixon, K. W., \& Broccoli, A. J. (2001). Anthropogenic warming of Earth's climate system. Science, 292(5515), 267-270. doi: 10.1126/science.1058154.

Lima, J. E. F. W. (2001). Recursos hídricos no Brasil e no mundo. Planaltina: Embrapa Cerrados. 46p.

Lima, M. A. (2002). Agropecuária brasileira e as mudanças climáticas globais: caracterização do problema, oportunidades e desafios. Cadernos de Ciência E Tecnologia, 19(3), 451-472. doi: 10.35977/0104-1096. cct2002.v19.8816.

Llambías, E. J. (2001). Geología de los cuerpos ígneos. Buenos Aires: Consejo Nacional de Investigaciones Científicas y Técnicas, Facultad de Ciencias Naturales e Instituto Miguel Lillo, Universidad Nacional de Tucumán. 234p.

Marengo, J. A. (2001). Mudanças climáticas globais e regionais: avaliação do clima atual do brasil e projeções de cenários climáticos do futuro. Rev. Bras. Meteorol., 16(1), 1-18.

Moreno, P. G., \& Freitas, P. L. de. (2009). Conhecendo a Terra: um olhar ecológico sobre o planeta. Rio de Janeiro: Pollux. 48p.

NOAA. National Oceanic and Atmospheric Administration. (2016). Peeling Back the Layers of the Atmosphere. NOAA. URL: https://www.nesdis.noaa.gov/ content/peeling-back-layers-atmosphere. Acesso 05.12 .2020

NOAA. National Oceanic and Atmospheric Administration. (2020a). Teacher Background: The Greenhouse Effect. NOAA. URL: https://www.esrl.noaa.gov/gmd/ education/info_activities/pdfs/TBI_greenhouse_effect.pdf. Acesso 05.12.2020.

NOAA. National Oceanic and Atmospheric Administration. (2020b). What is the carbon cycle? NOAA. URL: https://oceanservice.noaa.gov/facts/carbon-cycle. html. Acesso 05.12.2020.

NOAA. National Oceanic and Atmospheric Administration. (2020c). Trends in Atmospheric Carbon Dioxide. NOAA. URL:https://www.esrl.noaa.gov/gmd/ccgg/ trends/mlo.html. Acesso 05.12.2020.

NWS. National Weather Service. (2020a). The Atmosphere. NWS. URL: https://www.weather.gov/jetstream/atmos intro. Acesso 05.11.2020.

NWS. National Weather Service. (2020b). The Hydrologic Cycle. NWS. URL: https://www.weather.gov/jetstream/hydro. Acesso 05.11.2020.

Oliveira, G. S., Silva, N. F., \& Henriques, R. 2009. $M u$ danças climáticas: ensinos fundamental e médio. Brasília: MEC, SEB, MCT, AEB. 348p. (Col. Explorando o ensino)

Onça, D. S. (2014). O papel do vapor d'água no sistema climático. Periódico Eletrônico Fórum Ambiental da Alta Paulista, 10(8), 1-12. doi: 10.17271/198008271082014772.

Orozco-Centeno, W. P., Branch Bedoya, J. W., \& Jiménez-Builes, J. A. (2014). Classification of fine-grained igneous, sedimentary and metamorphic rocks through structured programming. Boletín de Ciencias de la Tierra, (36), 5-9. doi: 10.15446/rbct.n36.44037.
Pistone, K., Eisenman, I., \& Ramanathan, V. (2014). Observational determination of albedo decrease caused by vanishing Arctic sea ice. Proc. Natl. Acad. Sci., 111(9), 3322-3326. doi: 10.1073/pnas.1318201111.

Post, W. M., Peng, T. H., Emanuel, W. R., King, A. W., Dale, V. H., \& DeAngelis, D. L. (1990). The global carbon cycle. Am. Sci., 78(4), 310-326.

Qu, X., \& Hall, A. (2007). What controls the strength of snow-albedo feedback? J. Clim., 20(15), 3971-3981. doi: 10.1175/JCLI4186.1.

Reboita, M. S., Krusche, N., Ambrizzi, T., \& Rocha, R. P. da (2012). Entendendo o Tempo e o Clima na América do Sul. Terræ Didatica, 8(1), 34-50. doi: 10.20396/td.v8i1.8637425.

Reboita, M. S., Pimenta, A. P., \& Natividade, U. A. (2015). Influência da inclinação do eixo de rotação da Terra na temperatura do ar global. Terre Didatica, 11(2), 67-77. doi: 10.20396/td.v11i2.8640680.

Rincón, L. E. C., \& Gutiérrez, F. A. A. (2012). Dinámica del ciclo del nitrógeno y fósforo en suelos. Revista Colombiana de Biotecnología, 14(1), 285-295.

Rodrigues, C. M. (2017). Evaporação e evapotranspiração. In: Shaidian, S., Guimarães, C. R., \& Rodrigues, C. M. (Org.). Hidrologia agrícola. Évora: Universidade de Évora. p. 215-234.

Ross, J. L. S. (2005). Os fundamentos da geografia da natureza. In: Ross, J. L. S (Org.). Geografia do Brasil. São Paulo: Editora da Universidade de São Paulo (Edusp). p. 13-51

Salby, M. L. (1996). Fundamentals of Atmospheric Physics. USA: Academic Press. 627p.

Schellnhuber, H. J., Cramer, W., Nakicenovic, N., Wigley, T., \& Yohe, G. (Eds.). (2006). Avoiding Dangerous Climate Change. New York: Cambridge University Press. 406p.

Souza, M. F. L., Longhini, C. M., Silva, A. M., \& Lentini, C. A. D. (2012). Ciclo do Carbono: Processos Biogeoquímicos, Físicos e Interações entre Compartimentos na Baía de Todos os Santos. Rev. Virtual de Química, 4(5), 566-582. doi: 10.5935/19846835.20120044

Summons, R. E. (1993). Biogeochemical cycles. In: Engel, M. H., \& Macko, S. A. (Eds.). Organic Geochemistry. Boston: Springer. p. 3-21.

Teodoro, P. H. M., \& Amorim, M. C. C. T. (2008). Mudanças climáticas: algumas reflexões. Rev. Bras. Climatol., 3, 25-35. doi: 10.5380/abclima.v3i0.25405.

Tundisi, J. G. (2003). Ciclo hidrológico e gerenciamento integrado. Cien. Cult., 55(4), 31-33.

Vieira, R. F. (2017). Ciclo do nitrogênio em sistemas agrícolas. Brasília: Embrapa Meio Ambiente. URL: http://www.alice.cnptia.embrapa.br/alice/handle/ doc/1090589. Acesso 03.11.2020.

Wallace, J. M., \& Hobbs, P. V. (2007). Atmospheric Science: An Introductory Survey. Elsevier. 485p.

Yamasoe, M. A., \& Corrêa, M. P. (2016). Processos radiativos na atmosfera: Fundamentos. São Paulo: Oficina de Textos. 142p.

Ynoue, R. Y., Reboita, M. S., Ambrizzi, T., \& Silva, G. A. M. da (2017). Meteorologia: noções básicas. São Paulo: Oficina de Textos. 184p. 